I. Higuchi

Nagoya Math. J,

Vol. 57 (1975), 27-35

\title{
ON THE TRANSITIVE DOMINATION PRINCIPLE FOR CONTINUOUS FUNCTION-KERNELS
}

\author{
ISAO HIGUCHI
}

\section{Introduction}

One of the interesting problems in the potential theory for a functionkernel is to investigate the relation between the domination principle and the balayage principle.

$\mathrm{N}$. Ninomiya first proved the equivalence of the above two principles for a positive symmetric continuous kernel using the Gauss-Ninomiya variation (cf. [6]).

For a positive non-symmetric lower semi-continuous kernel, M. Kishi established a new existence theorem and proved in [4] that a kernel $G$ satisfies the domination principle if and only if $G$ satisfies the balayage principle under the additional condition that $G$ and its adjoint $\breve{G}$ satisfy the continuity principle.

For a continuous function-kernel (in the extended sense), it is well known that a kernel $G$ itself satisfies the continuity principle when $G$ satisfies the domination principle. Does the adjoint $\check{G}$ satisfy the continuity principle? M. Itô and the author proved in [2] that $\breve{G}$ also satisfies the continuity principle when $G$ satisfies the domination principle. We used there a result obtained in [3] where M. Itô developed the theory of generalized kernels by means of elementary kernels and resolvents.

Let $G$ and $N$ be continuous function-kernels. In this paper we shall prove that $G$ satisfies the relative domination principle with respect to $N$ if and only if $\check{G}$ satisfies the transitive domination principle with respect to $\Sigma$. The analogous result is first obtained by M. Kishi under the additional condition that $G$ and $\breve{G}$ satisfy the continuity principle (cf. [4]).

Our result shall give a simple proof of the theorem obtained in [2] that $G$ satisfies the domination principle if and only if $\breve{G}$ satisfies the

Received May 14, 1974. 
domination principle. Therefore the continuity principle for $\breve{G}$ follows immediately from the domination principle for $G$. It will be also shown that $G$ satisfies the maximum principle if and only if $G$ satisfies the positive mass principle. All the proofs depend only on the existence theorem of Kishi.

\section{Definitions}

Let $X$ be a locally compact Hausdorff space. A non-negative function $G(x, y)$ on $X \times X$ is called a continuous function-kernel on $X$ if $G(x, y)$ is continuous in the extended sense on $X \times X$, finite except for the diagonal set of $X \times X$ and $0<G(x, x) \leqq+\infty$ for any $x \in X$. The kernel $\breve{G}$ defined by $\breve{G}(x, y)=G(y, x)$ is called the adjoint kernel of $G$. The potential $G \mu(x)$ and the adjoint potential $G \mu(x)$ of a positive Radon measure $\mu$ in $X$ are defined by

$$
G \mu(x)=\int G(x, y) d \mu(y) \text { and } \check{G} \mu(x)=\int \check{G}(x, y) d \mu(y)
$$

respectively.

The $G$-energy of $\mu$ is defined by $\int G \mu(x) d \mu(x)$. We denote by $M_{0}$ the family of all positive measures with compact support and by $E_{0}=E_{0}(G)$ the family of all measures in $M_{0}$ with finite $G$-energy. Evidently $E_{0}(G)$ $=E_{0}(\breve{G})$.

We say that a property holds G-p.p.p. on a subset $A$ of $X$ if it holds on $A$ except a set of the inner $\nu$-measure 0 for every $\nu \in E_{0}$.

\section{Existence theorem of Kishi and its modification}

In the succeeding sections, the following existence theorem plays a fundamental role.

EXISTENCE THEOREM OF KISHI ([1], [5]). Let $K$ be a compact Hausdorff space, $G$ be a finite continuous function-kernel on $K$ and $u(x)$ be a non-negative finite continuous function on $K$. Then there exists a measure $\lambda$ in $M_{0}$ such that

$$
\begin{array}{ll}
G \lambda(x) \geqq u(x) & \text { on } K, \\
G \lambda(x)=u(x) & \text { on } S \lambda .
\end{array}
$$

First we modify the above existence theorem into the following form. 
LEMMA 1. Let $G$ be a continuous function-kernel on a locally compact Hausdorff space $X, K$ be a compact subset of $X$ and $u(x)$ be a nonnegative finite continuous function on $K$. Take a sequence $\left\{G_{n}\right\}$ of finite continuous function-kernels such that $G_{n} / G$. Then there exists a vaguely bounded sequence $\left\{\lambda_{n}\right\}$ of positive measures supported by $K$ satisfying

$$
\begin{array}{ll}
G_{n} \lambda_{n}(x) \geqq u(x) & \text { on } K, \\
G_{n} \lambda_{n}(x)=u(x) & \text { on } S \lambda_{n} .
\end{array}
$$

And a vague cluster point $\lambda$ of $\left\{\lambda_{n}\right\}$ satisfies

$$
\begin{aligned}
& G \lambda(x) \geqq u(x) \quad \nu \text {-a.e. on } K \text { for every } \nu \in C(\check{G} ; K) \text {, } \\
& G \lambda(x) \leqq u(x) \quad \text { on } S \lambda,
\end{aligned}
$$

where $C(\check{G} ; K)$ denotes the family of all measures in $M_{0}$ such that $\breve{G}_{\nu}(x)$ is finite continuous on $K$.

Proof. Let $G^{\prime}$ be the restriction of $G$ to $K \times K$. Then $G^{\prime}$ is evidently a continuous function-kernel on $K$ and $G^{\prime} \mu(x)=G \mu(x)$ on $K$ for any positive Radon measure $\mu$ in $K$. Therefore we have only to prove it in the case that $X=K$.

By the existence theorem of Kishi, there exists for any $n$, a measure $\lambda_{n} \in M_{0}$ satisfying

$$
\begin{array}{ll}
G_{n} \lambda_{n}(x) \geqq u(x) & \text { on } K, \\
G_{n} \lambda_{n}(x)=u(x) & \text { on } S \lambda_{n} .
\end{array}
$$

The strictly positiveness of $G$ on the diagonal set asserts that $\left\{\lambda_{n}\right\}$ is vaguely bounded. Therefore a subnet $\left\{\lambda_{\omega} ; \omega \in D, D\right.$ is a directed set $\}$ of the sequence $\left\{\lambda_{n}\right\}$ converges vaguely to a positive measure $\lambda$ supported by $K$. $\lambda$ fulfils (3) and (4). In fact, for any $\nu \in C(\check{G} ; K)$, we have

$$
\begin{aligned}
\int G \lambda d \nu & =\int \check{G} \nu d \lambda=\lim _{\omega} \int \check{G} \nu d \lambda_{\omega}=\lim _{\omega} \int G \lambda_{\omega} d \nu \\
& \geqq \varlimsup_{\omega} \int G_{\omega} \lambda_{\omega} d \nu \geqq \int u d \nu,
\end{aligned}
$$

hence (3). Let $\omega_{0} \in D$ and $x_{0} \in S \lambda$ be fixed. There exists a net $\left\{x_{\omega}\right\}$ converging to $x_{0}$ with $x_{\omega} \in S \lambda_{\omega}$. Then we obtain

$$
\begin{aligned}
G_{\omega_{0}} \lambda\left(x_{0}\right) & =\lim _{\omega} G_{\omega_{0}} \lambda_{\omega}\left(x_{\omega}\right) \leqq \underline{\lim } G_{\omega} \lambda_{\omega}\left(x_{\omega}\right) \\
& \leqq \lim _{\omega} u\left(x_{\omega}\right)=u\left(x_{0}\right)
\end{aligned}
$$


ISAO HIGUCHI

hence (4).

Remark 1. The analogous result holds for a lower semi-continuous function-kernel $G$ and a bounded upper semi-continuous function $u(x)$.

\section{Relative domination principle and transitive domination principle}

Let us start with the definitions of principles.

( I ) Continuity principle: A kernel $G$ is said to satisfy the continuity principle when, for any $\mu \in M_{0}$, the finite continuity of the restriction of $G \mu(x)$ to $S \mu$ implies the finite continuity of $G \mu(x)$ on the whole space $X$.

(II) Relative domination principle: $G$ is said to satisfy the relative domination principle with respect to $N$ (written simply $G \prec N$ ) when, for $\mu \in E_{0}(G)$ and $\nu \in M_{0}$, an inequality $G \mu(x) \leqq N \nu(x)$ on $S \mu$ implies the same inequality on $X$. In the case that it holds only when $\nu=\varepsilon_{x_{0}}$ for any $x_{0} \in C S \mu$, we call it the elementary relative domination principle.

(III) Transitive domination principle: We say that $G$ satisfies the transitive domination principle with respect to $N$ (written simply $G \square N$ ) when, for $\mu$ and $\nu$ in $M_{0}$ with $S \mu \cap S \nu=\phi$, an inequality $G \mu(x) \leqq G \nu(x)$ on $S \mu$ implies the inequality $N \mu(x) \leqq N \nu(x)$ on $X$.

Remark 2. It is well known, for strictly positive continuous functionkernels $G$ and $N$, that $G$ satisfies the continuity principle if $G$ satisfies the elementary relative domination principle with respect to $N$. This is true for our continuous function-kernels. In fact, let $x_{0}$ be any nonisolated point in $X$. Then there exist a point $y_{0}\left(y_{0} \neq x_{0}\right)$ and an open neighbourhood $V\left(x_{0}\right)$ of $x_{0}$ such that $N\left(x, y_{0}\right)>0$ for any $x \in V\left(x_{0}\right)$, because $N\left(x_{0}, x_{0}\right)>0$. Consequently, the continuity principle for $G$ follows by the same way as in the case of strictly positive kernels.

Remark 3. If $\check{G}$ satisfies the continuity principle, Lusin's theorem asserts that the inequality (3) in Lemma 1 is equivalent to the following inequality $\left(3^{\prime}\right)$.

$$
G \lambda(x) \geqq u(x) \quad G \text {-p.p.p. on } K .
$$

LEMMA 2. Let $G$ and $N$ be continuous function-kernels on $X$. Suppose that $\breve{G}$ satisfies the transitive domination principle with respect to Ň. Then $G$ satisfies the continuity principle. 
Proof. It suffices to prove that $G$ satisfies the elementary relative domination principle with respect to $N$ (cf. Remark 2).

Suppose that for $\mu \in E_{0}(G)$ and $x_{0} \in C S \mu$, an inequality $G \mu(x) \leqq N \varepsilon_{x_{0}}(x)$ holds on $S \mu$. We shall show that $G \mu(y) \leqq N \varepsilon_{x_{0}}(y)$ holds for any $y \in C S \mu$. By Lemma 1 , there exists a vaguely bounded sequence $\left\{\varepsilon_{y n}^{\prime}\right\}$ of positive measures supported by $S \mu$ satisfying

$$
\check{G}_{n} \varepsilon_{y n}^{\prime}(x) \geqq \check{G} \varepsilon_{y}(x) \quad \text { on } S \mu
$$

and

$$
\check{G}_{n} \varepsilon_{y n}^{\prime}(x)=\check{G}_{y}(x) \quad \text { on } S \varepsilon_{y n}^{\prime},
$$

because $\check{G}_{\varepsilon}(x)$ is finite continuous on $S \mu$. A vague cluster point $\varepsilon_{y}^{\prime}$ of $\left\{\varepsilon_{y n}^{\prime}\right\}$ fulfils

$$
\check{G} \varepsilon_{y}^{\prime}(x) \leqq \check{G} \varepsilon_{y}(x) \quad \text { on } S \varepsilon_{y}^{\prime} \subset S \mu
$$

$\check{G} \square \check{N}$ asserts

$$
\check{N} \varepsilon_{y}^{\prime}(x) \leqq \check{N} \varepsilon_{y}(x) \quad \text { on } X
$$

Then we obtain

$$
\begin{aligned}
G \mu(y) & =\int G \mu(x) d \varepsilon_{y}(x)=\int \check{G} \varepsilon_{y} d \mu \leqq \int \check{G}_{n} \varepsilon_{y n}^{\prime} d \mu \\
& \leqq \int G \mu d \varepsilon_{y n}^{\prime} \leqq \int N \varepsilon_{x_{0}} d \varepsilon_{y n}^{\prime} \quad \text { for any } n
\end{aligned}
$$

$\varepsilon_{y}^{\prime}$ being a vague cluster point of $\left\{\varepsilon_{y n}^{\prime}\right\}$, we have

$$
\begin{aligned}
G \mu(y) & \leqq \int N \varepsilon_{x_{0}} d \varepsilon_{y}^{\prime}=\int \check{N} \varepsilon_{y}^{\prime} d \varepsilon_{x_{0}} \\
& \leqq \int \check{N} \varepsilon_{y} d \varepsilon_{x_{0}}=N \varepsilon_{x_{0}}(y) .
\end{aligned}
$$

Consequently $G$ satisfies the elementary relative domination principle with respect to $N$.

THEOREM 1. Let $G$ and $N$ be continuous function-kernels on a locally compact Hausdorff space $X$. Then the following statements are equivalent.

(a) $G$ satisfies the relative domination principle with respect to $N$.

(b) $G$ satisfies the elementary relative domination principle with respect to $N$.

(c) Ğ satisfies the transitive domination principle with respect to $\check{N}$. 
Proof. Evidently (a) $\rightarrow$ (b).

(b) $\rightarrow$ (c). Suppose that for $\mu$ and $\nu$ in $M_{0}$ with $S \mu \cap S \nu=\phi$, an inequality $\breve{G} \mu(x) \leqq \breve{G} \nu(x)$ holds on $S \mu$. We have only to obtain the inequality $\check{N}_{p} \mu(y) \leqq \check{N} \nu(y)$ for any positive number $p$ and for any $y \in X$, where $\check{N}_{p}=\check{N}_{p}(x, y)=\inf \{\check{N}(x, y), p\}$. By Lemma 1 , there exists a sequence $\left\{\varepsilon_{y p n}^{\prime}\right\}$ of measures in $E_{0}(G)$ supported by $S \mu$ satisfying

$$
G_{n} \varepsilon_{y p m}^{\prime}(x) \geqq N_{p} \varepsilon_{y}(x) \quad \text { on } S \mu
$$

and

$$
G_{n} \varepsilon_{y p n}^{\prime}(x)=N_{p} \varepsilon_{y}(x) \quad \text { on } S \varepsilon_{y p n} .
$$

A vague cluster point $\varepsilon_{y p}^{\prime}$ of $\left\{\varepsilon_{y p n}^{\prime}\right\}_{n=1}^{\infty}$ fulfils

$$
G \varepsilon_{y p}^{\prime}(x) \leqq N_{p} \varepsilon_{y}(x) \leqq N \varepsilon_{y}(x) \quad \text { on } S \varepsilon_{y p}^{\prime} \subset S \mu .
$$

(b) implies

$$
G \varepsilon_{y p}^{\prime}(x) \leqq N \varepsilon_{y}(x) \quad \text { on } X
$$

Then

$$
\begin{aligned}
\check{N}_{p} \mu(y) & =\int \check{N}_{p} \mu d \varepsilon_{y}=\int N_{p} \varepsilon_{y} d \mu \leqq \int G_{n} \varepsilon_{y p n}^{\prime} d \mu \\
& \leqq \int \check{G}_{\mu} d \varepsilon_{y p n}^{\prime} \leqq \int \check{G}_{\nu} d \varepsilon_{y p n}^{\prime} \quad \text { for any } n .
\end{aligned}
$$

And $\varepsilon_{y p}^{\prime}$ being a vague cluster point of $\left\{\varepsilon_{y m n}^{\prime}\right\}_{n=1}^{\infty}$, we obtain

$$
\begin{aligned}
N_{p} \mu(y) & \leqq \int \check{G} \nu d \varepsilon_{y p}^{\prime}=\int G \varepsilon_{y p}^{\prime} d \nu \\
& \leqq \int N \varepsilon_{y} d \nu(y)=\check{N} \nu(y) .
\end{aligned}
$$

(c) $\rightarrow$ (a). Assume that $G \mu(x) \leqq N \nu(x)$ holds on $S \mu$ for $\mu \in E_{0}$ and $\nu \in M_{0}$. By virtue of Lemma 2, (c) asserts that $G$ satisfies the continuity principle and so we can find, for any $n$ and any $y \in C S \mu$, a measure $\tilde{\varepsilon}_{y n} \in E_{0}$ supported by $S \mu$ satisfying

$$
\check{G} \tilde{\varepsilon}_{y n}(x) \geqq \check{G}_{n} \varepsilon_{y}(x) \quad \text { G-p.p.p. on } S \mu
$$

and

$$
\check{G} \tilde{\varepsilon}_{y n}(x) \leqq \check{G}_{n} \varepsilon_{y}(x) \leqq \check{G} \varepsilon_{y}(x) \quad \text { on } S \tilde{\varepsilon}_{y n}
$$

(cf. (3') in Remark 3). (c) implies 


$$
\check{N} \tilde{\varepsilon}_{y n}(x) \leqq \check{N} \varepsilon_{y}(x) \quad \text { on } X .
$$

Consequently we obtain

$$
\begin{aligned}
G_{n} \mu(y) & =\int \check{G}_{n} \varepsilon_{y} d \mu \leqq \int \check{G} \tilde{\varepsilon}_{y n} d \mu \leqq \int G \mu d \tilde{\varepsilon}_{y n} \\
& \leqq \int N \nu d \tilde{\varepsilon}_{y n} \leqq \int \check{N} \tilde{\varepsilon}_{y n} d \nu \leqq \int \check{N} \varepsilon_{y} d \nu=N \nu(y)
\end{aligned}
$$

Letting $n$ tend to $+\infty$, we have $G \mu(y) \leqq N \nu(y)$. This completes the proof.

Remark 4. M. Kishi proved this theorem under the additional condition that $G$ and $\check{G}$ satisfy the continuity principle. But by the above argument this condition can be omitted.

\section{Domination principle and continuity principle}

As an application of Theorem 1, we consider the domination, the continuity and the balayage principles for a continuous function-kernel $G$ and its adjoint $\check{G}$.

(IV) Domination principle: For $\mu \in E_{0}$ and $\nu \in M_{0}$, an inequality $G \mu(x) \leqq G \nu(x)$ on $S \mu$ implies the same inequality on $X$. Especially we call it the elementary domination principle when $\nu=\varepsilon_{x_{0}}$ for any $x_{0} \in C S \mu$.

(V) Balayage principle: For a given compact set $K$ and a given measure $\mu$ in $M_{0}$, there exists a measure $\mu^{\prime}$ in $M_{0}$ supported by $K$ such that

$$
\begin{array}{ll}
G \mu^{\prime}(x)=G \mu(x) & G \text {-p.p.p. on } K, \\
G \mu^{\prime}(x) \leqq G \mu(x) & \text { on } X .
\end{array}
$$

THEOREM 2. Let $G$ be a continuous function-kernel on $X$. Then both $G$ and $\check{G}$ satisfy the continuity principle if either $G$ or $\breve{G}$ satisfies the domination principle.

Proof. We have only to prove that $\breve{G}$ satisfies the continuity principle when $G$ satisfies the domination principle. The domination principle for $G$ implies that $G \prec G$ and also implies, by virtue of Theorem 1, that $\check{G} \square \breve{G}$. Therefore $\breve{G}$ satisfies the elementary domination principle and, by Remark $2, \breve{G}$ satisfies the continuity principle. 
THEOREM 3. Let $G$ be a continuous function-kernel on $X$. Then $G$ satisfies the domination principle if and only if $\breve{G}$ does.

Proof. By Theorem 1, $G \prec G$ implies that $\check{G} \square \check{G}$ and therefore $\check{G}$ satisfies the elementary domination principle. The equivalence of (a) and (b) in Theorem 1 asserts that $\check{G}$ satisfies the domination principle. The converse is also true.

Similarly the following theorem of Kishi is an immediate consequence of Theorem 2 and Remark 3.

THEOREM 4. Let $G$ be a continuous function-kernel on $X$. Then $G$ satisfies the balayage principle if and only if $G$ satisfies the domination principle.

Remark 5. Theorem 3 and Theorem 4 were first proved by M. Kishi in [4] under the condition that $G$ and $\breve{G}$ satisfy the continuity principle and were proved by M. Itô and the author in [2] without that condition using a result of generalized kernels established by M. Itô in [3]. The above argument gives a simple proof depending only on Theorem 1 .

\section{Maximum principle and positive mass principle}

Finally we investigate the relation between the maximum and the positive mass principle.

( VI ) Maximum principle: For $\mu \in M_{0}$, an inequality $G \mu(x) \leqq 1$ on $S \mu$ implies the same inequality on $X$.

(VII) Complete maximum principle: For $\mu \in E_{0}, \nu \in M_{0}$ and for a non-negative number $a$, an inequality $G \mu(x) \leqq G \nu(x)+a$ on $S \mu$ implies the same inequality on $X$.

(VIII) Positive mass principle: For $\mu$ and $\nu$ in $M_{0}$ with $S \mu \cap S \nu$ $=\phi$, an inequality $G \mu(x) \leqq G \nu(x)$ on $S \mu$ implies the inequality $\int d \mu \leqq \int d \nu$.

THEOREM 5. Let $G$ be a continuous function-kernel on $X$. Then $G$ satisfies the maximum principle if and only if $\check{G}$ satisfies the positive mass principle.

Proof. The maximum principle for $G$ implies that $G \prec 1$, where 1 is the constant kernel. By theorem $1, G \prec 1$ if and only if $\check{G} \square \check{1}=1$. $\check{G} \square 1$ is just the positive mass principle for $\check{G}$. 
By this theorem, we have the following corollaries without assuming that $G$ and $\check{G}$ satisfy the continuity principle.

CoRollary 1. Let $G$ be a continuous function-kernel on $X$. Then $G$ satisfies the complete maximum principle if and only if $G$ satisfies the domination principle and the maximum principle.

CoRollary 2. Let $G$ and $N$ be continuous function-kernels on $X$ such that $G$ satisfies the relative domination principle with respect to $N$. Then $G$ satisfies the maximum principle if $N$ does.

Proof. It is sufficient to prove that $\check{G}$ satisfies the positive mass principle. Suppose that $\check{G} \mu(x) \leqq \breve{G} \nu(x)$ on $S \mu$ for $\mu$ and $\nu$ in $M_{0}$ with $S_{\mu} \cap S_{\nu}=\phi . \quad G \prec N$ implies, by Theorem 1, that $\check{G} \square \check{N}$. Consequently we have $\check{N} \mu(x) \leqq \check{N} \nu(x)$ on $X$. On the other hand $N \prec 1$ asserts, by Theorem 5, that $\check{N} \square 1$ and hence that $\int d \mu \leqq \int d \nu$. Therefore $G$ satisfies the positive mass principle.

\section{REFERENCES}

[1] R. Durier: Thèse, Faculté des Sciences d'Orsay, Université de Paris, 1969.

[2] I. Higuchi and M. Itô: On the theorem of Kishi for a continuous function-kernel, Nagoya Math. J., 53 (1974), p. 127-135.

[ 3 ] M. Itô: Sur les principes divers du maximum et le type positif, Nagoya Math. J., 44 (1971), p. 133-164.

[4] M. Kishi: Maximum principles in the potential theory, Nagoya Math. J., 23 (1963), p. 165-187.

[ 5 ] M. Kishi: An existence theorem in potential theory, Nagoya Math. J., 27 (1966), p. $133-137$.

[6] N. Ninomiya: Etude sur la théorie du potentiel pris par rapport au noyau symétrique, J. Inst. Polytechnics, Osaka City Univ., 8 (1957), p. 147-178.

Department of Mathematics

Faculty of General Education

Aichi Institute of Technology 